\title{
Electrocardiographic Imaging and Phase Mapping Approach for Atrial Fibrillation: A Simulation Study
}

\author{
Rémi Dubois ${ }^{1,2}$, Ali Pashaei ${ }^{1,3}$, Josselin Duchateau ${ }^{1,2}$, Edward Vigmond ${ }^{1,3}$ \\ ${ }^{1}$ Electrophysiology and Heart Modeling Institute (IHU LIRYC), University of Bordeaux, \\ ${ }^{2}$ CRCTB Inserm U1045, Bordeaux University Hospital (CHU), \\ Electrophysiology and Ablation Unit, Bordeaux, France. \\ ${ }^{3}$ Bordeaux Institute of Mathematics UMR 5251, University of Bordeaux, France
}

\begin{abstract}
Objectives: We aimed to evaluate the non-invasive phase mapping methods used in clinical practice on atrial signals during atrial fibrillation (AF). Methods: A modified Courtemanche human atrial ionic model was used to run AF simulations. Extracellular potentials on the epicardium were computed and propagated to the body surface through a homogeneous torso conductor using the Boundary Element Method. The obtained body surface potentials were sampled in 252 different locations to replicate clinical recordings. The clinical non-invasive AF mapping workflow was then applied to this body surface data to reconstruct atrial epicardial potentials and corresponding phase signals. Results: The AF cycle lengths were well estimated for the two datasets (mean relative error magnitude $M R E=5.4 \%$ and $3.8 \%$ for the two simulation sequences with no noise). Results were maintained when up to $10 \mathrm{~dB}$ of signal noise on the body surface recordings or $7.5+/-3.4 \mathrm{~mm}$ geometrical noise on the electrode locations were added. The phase locking values (PLV) were 0.62 and 0.78 respectively for the two simulation sequences, indicating a fair correlation between the phase signals. Regions showing reentries were correctly localized. Reconstructed phase singularity positions were insensitive to added electrical and geometrical noise.
\end{abstract}

\section{Introduction}

Recent developments in body surface mapping and computer processing have allowed non-invasive mapping of atrial activation during cardiac arrhythmias with increasingly finer resolution. We developed a noninvasive atrial fibrillation (AF) mapping workflow that combines Electrocardiographic Imaging (ECGi) and phase mapping to localize reentry and focal activation during on-going AF. While some studies have shown the efficiency of the method in clinical practice [1-3], it remains challenging to determine the quantitative accuracy of such an approach, as validation data have so far been inaccessible in human and animal models. Conversely, some recent studies have compared phase mapping in atria to other mapping techniques and have identified certain limitations [4-6].

Hence, the aim of this study was to evaluate the accuracy of the features that are used to guide AF ablation in clinical practice. To achieve this goal, we used simulated data for which a clear gold standard is available. We simulated AF epicardial data with a realistic atrial ionic model. The computed signals were then propagated to the torso to obtain body surface potential maps from 252 simulated recording locations. Our non-invasive AF mapping workflow that includes the inverse computation of electrical potentials and corresponding phase followed by feature extraction was applied to each simulated dataset. Feature values were compared to those obtained directly from the transmembrane potentials.

\section{Methods}

\subsection{Computer model of AF}

Computational model of the heart

A finite element bilayer model of the atria composed of $360 \mathrm{~K}$ nodes and incorporating fibre orientation was constructed. Transmembrane ionic activity was modeled using the Courtemanche human atrial myocyte model. Fibrosis was added to the model using late gadolinium enhanced MRI data from patient MRI. Pixel intensity was normalized and then used as a probability for assigning scar properties to mesh nodes under corresponding pixels. Scar nodes were considered nonconductive and simply removed from the mesh. Computations were performed on both the fibrotic/structurally diseased model (AFSD) as well as the original non-fibrotic (AFnSD) version. 
Reentry was initiated by a S1S2 protocol.

\section{Torso Potentials}

To compute torso potentials, a quasi-static boundary element formulation was used with a zero-flux constraint on the torso surface. The torso geometry was described by 9800 triangular boundary elements with between-node linear interpolation. Free space potentials were computed on the torso surface using atrial transmembrane currents, and the surface potentials solved using singular value decomposition. The torso model used in this study was a homogenous torso, with no blood in the chambers and no ventricles.

\section{Numerical scheme for adding noise in the data}

Two noisy sequences were derived from each simulated sequence (AFSD and AFnSD): one (EE) with $10 \mathrm{~dB}$ of white noise added to the body surface potentials, and one $(\mathrm{PN})$ with non correlated noise on each body surface electrode positions (mean: 7.5+/-3.4mm).

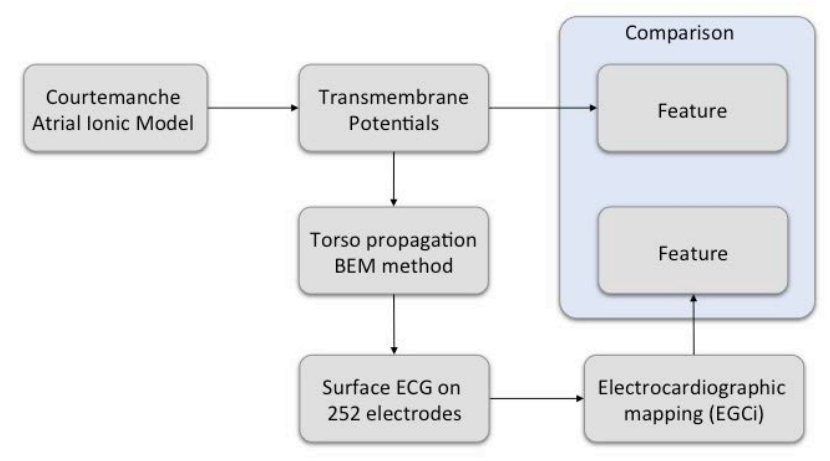

Figure 1: Study workflow. Simulated data are generated from an atrial model producing transmembrane potentials and body surface ECGs. Each feature is computed 1) directly on the transmembrane potentials (gold standard), and 2) on the reconstructed epicardial signals after inverse computation.

\subsection{Non-invasive AF mapping}

The Method of Fundamental Solutions (MFS) was used to solve the inverse problem and compute epicardial potentials [7]. Briefly, the electrical activity of the heart is assumed to propagate homogenously through the torso considered as a passive medium. The forward transfer matrix that links the heart sources to 252 measuring electrodes on the body surface is obtained via the fundamental solution of the Laplace's equation expressed between virtual sources around the heart and torso and with the boundary conditions on the body surface. Being a meshless method, the MFS method has a reduced computational workload and mesh production time compared to other tradition methods. The transfer matrix is ill-conditioned, yielding a noise-sensitive inverse solution. For this reason, a standard L2 optimization process with a Tikhonov regularization was used to compute the MFS coefficients. Regularization parameter value was computed using the composite residual and smoothing operator (CRESO) method [8].

\subsection{Features for atrial fibrillation}

\section{Feature Preprocessing}

In human AF, atrial myocytes depolarize every 120 to $250 \mathrm{~ms}$. This AF cycle length (AFCL) may vary from one atrium to the other or within a given chamber. Noninvasively reconstructed electrograms (rEGM) are sensitive to electrical noise, hence a non-recursive band pass frequency filter between $4-8 \mathrm{~Hz}$ (i.e. with linear phase) was applied to all atrial signals to enhance the frequencies of interest.

\section{Phase Signals, and Singularity Maps}

Phase signals were computed using the Hilbert transform on the filtered rEGM. Phase maps were derived from the phase signals by plotting the instantaneous phase values on the atria geometry. Instantaneous Phase Singularities (PS) were defined as locations in the phase maps around which phase values spanned between $-\square$ and $\square$. This specific fingerprint has been demonstrated to correspond to reentrant patterns of the activation wavefront [9]. The standard approach to detect SP at node $X_{j}$ is to compute an integral over a close loop $C_{X}$ around this point. In our study, the discrete form of the integral was approximated by the sum around the nodes $X_{j}$ in the atrial mesh:

$$
S_{X_{j}}=\oint_{C_{Y j}} d l \nabla \theta_{X j}=\sum_{i \in C_{X i}} \theta_{X i+1}-\theta_{X i}
$$

Where $\square \mathrm{xi}$ is the phase value of node $X_{i}$. Hence, a phase singularity is present in $X_{j}$ if the value of $S_{X j}$ equals $\pm 2 \pi$. Phase singularity maps were then obtained by averaging the values of $S_{X}$ over time:

$$
\bar{S}_{X}=\frac{1}{T} \sum_{t=1 T}\left|S_{X}(t)\right|
$$

where $T$ is the duration of the AF episode.

\subsection{Data analysis}

AFCL, Phase Signals and Phase Singularity Maps were computed over 6 simulated sequences. Three on the non-structural diseased model (AFnSD): no noise, with $10 \mathrm{db}$ of electrical noise and with electrode position error; and three on the structural diseased model (AFSD). Each simulation led to 1) the transmembrane potentials that served as a gold standard and 2) the rEGMs that corresponded to the output of the inverse problem (Figure 1). 
The accuracy of the AFCL maps was quantified by measuring the magnitude of the relative error for each node and was reported as the average value over all nodes.

$$
\varepsilon^{A F C L}=\frac{1}{N} \sum_{X_{i}} \varepsilon_{X_{i}}^{A F C L}=\frac{1}{N} \sum_{X_{i}}\left|\overline{C L}_{X_{i}}-\overline{C L}_{X_{i}}^{r e f}\right|
$$

where $N$ is the number of nodes in the atrial mesh, $\overline{C L}_{X_{i}}$ (resp. $\overline{C L}_{X_{i}}^{r e f}$ ) is the mean AFCL measured on the reconstructed phase signal at node $X_{i}$ (resp. on the transmembrane potentials).

The accuracy of the phase signals for each node was quantified using the Phase Locking Value [10]. This index estimates the synchronicity between two signals whatever the constant phase shift between them, it was defined for the node $X_{i}$ as:

$$
P L V_{X_{i}}=\frac{1}{T} \mid \sum_{t} \exp \left(j\left(\theta_{X_{i}}^{r e f}(t)-\theta_{X_{i}}(t)\right)\right)
$$

where $\theta_{X_{i}}^{r f}(t)-\theta_{X_{i}}(t)$ is the difference between the gold standard value of the phase and the phase computed on the rEGM for node $X_{i}$ at time $t$. PLV equals 1 when the two signals differ in phase only by a constant, and equals 0 if they are non-correlated.

The correspondence between the phase singularity maps was quantified using the Correlation Coefficient (CC), where $\square_{\mathrm{s}}$ is the spatial average of $S$.:

$$
C C=\frac{\sum_{X}\left(\bar{S}_{X}-\mu_{\bar{S}}\right)\left(\bar{S}_{X}^{- \text {ref }}-\mu_{S^{\text {rf }}}\right)}{\left\|\bar{S}_{X}-\mu_{S}\left|\left\|||_{X}^{\text {ref }}-\mu_{\bar{S}^{n f}}\right\|\right.\right.}
$$

\section{Results}

\subsection{AF cycle length}

Table 1, column 1 shows the correspondence between the AFCLs, indicating a good accuracy across all the simulated sequences. Errors slightly increased with noise but not substantially. Figure 2 shows the distribution of the AFCL error for AFnSD-NN. Mean relative errors are substantially smaller in the left atrium compared to the right atrium. This difference is observed across all simulated sequences.

\subsection{Phase locking values}

Table 1, columns 2 shows the Phase Locking Values for each simulated sequence. Overall synchronization of the phase signals computed from the body surface data and gold standard potentials was satisfactory. The spatial distribution of the PLV over the 6 simulated sequences is presented in Figures 3. It indicates that the phase signals are better reconstructed in the left atria and lateral right atrial wall compared to the coronary sinus ostium.

Table 1. Errors in AFCLs, and Phase Locking Values

\begin{tabular}{rrr}
\hline & \multicolumn{1}{c}{$\square_{\mathrm{r}}(\mathrm{ms})$} & PLV \\
& {$\left[1^{\text {st }}-3^{\text {rd }}\right.$ quartile $]$} & \\
\hline Normal Heart (AFnSD) & & \\
No noise (NN) & $6.13[1.9-18.0]$ & $0.69[0.45-0.87]$ \\
10db Electrical noise (EN) & $6.18[1.9-18.0]$ & $0.69[0.44-0.87]$ \\
Electrode position noise (PN) & $6.00[2.0-18.5]$ & $0.67[0.53-0.86]$ \\
Structurally diseased Heart (AFSD) & & \\
No noise (NN) & $4.48[2.1-9.2]$ & $0.77[0.61-0.88]$ \\
10db Electrical noise (EN) & $4.58[2.0-9.2]$ & $0.77[0.60-0.87]$ \\
Electrode position noise (PN) & $5.00[2.2-10.4]$ & $0.77[0.58-0.87]$ \\
\hline
\end{tabular}

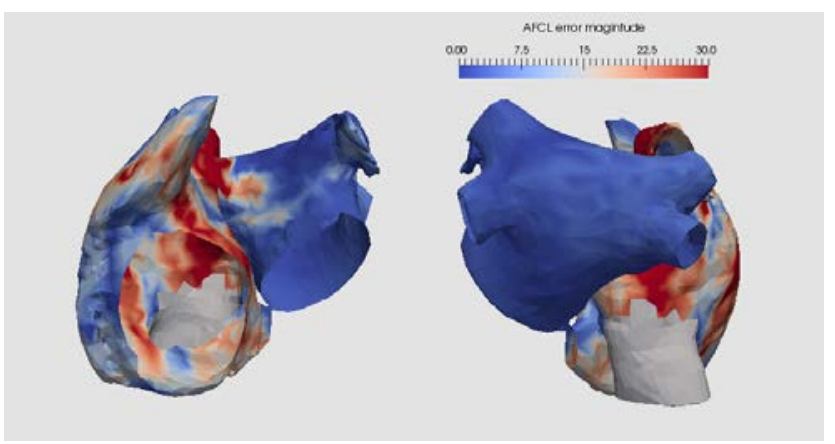

Figure 2: Error magnitude in AF Cycle Length. Results are better in the Left Atria compare to the Right Atria

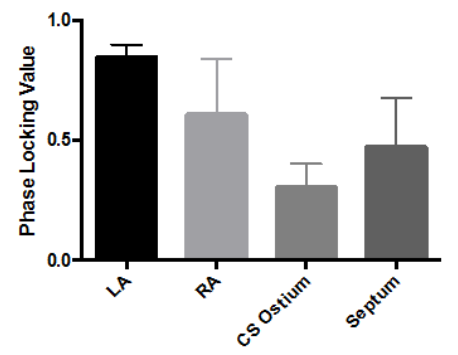

Figure 3: Spatial distribution of the Phase Locking Values for the 6 simulated sequences

\section{3. $\quad$ Singularity maps}

The CC between the computed singularity maps and the reference maps are poor (AFnSHD: No noise: 0.11(0.27), EE: 0.09, PN 0.06; AFSHD: No noise: 0.27, EE: 0.25, PN 0.22) (Figure 3); CCs decrease when noise is added to the input signal or the electrode positions. Figure 4 shows the SP maps for the AFSHD sequence. The upper panel corresponds to the map computed directly from the transmembrane potentials (gold standard) and the lower panel to the one obtained when electrode position error is added in the sequence. 'Hot spot' locations (posterior left inferior pulmonary vein, 
and anterior left atrium) are well identified. We notice a false positive in the nearby anterior right septum.

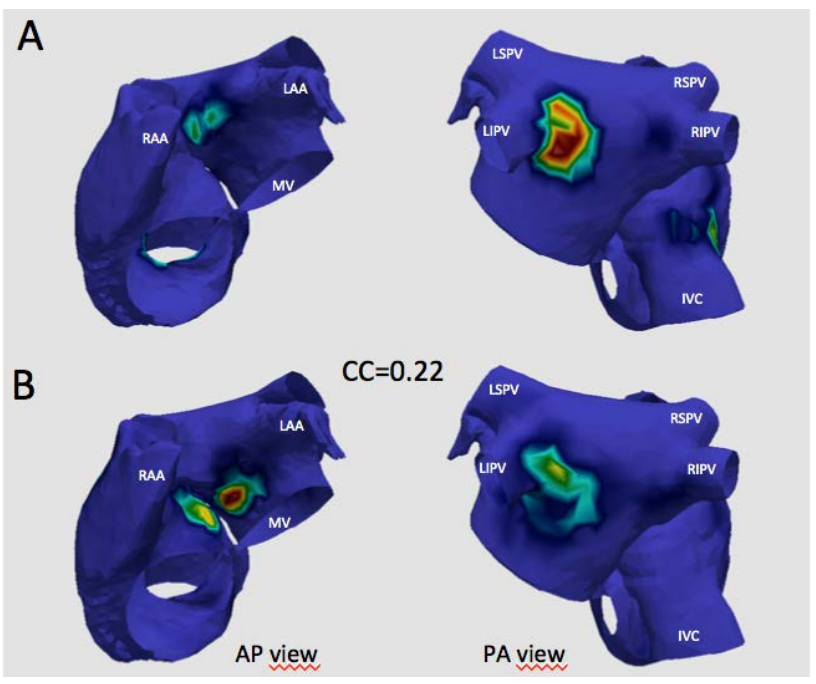

Figure 4: Singularity maps (A) computed on the transmembrane potentials (gold standard); and (B) on the reconstructed signals (rEGM). The CC between these two maps is also indicated.

\section{Discussion}

The spatial distribution of the AF cycle length and the The spatial distribution of AF cycle length and the location of phase singularities are useful features to target ablation. We showed in this study that these can be estimated using a combined approach of ECGi and phase mapping. However, the poor values of CCs between singularity maps indicate that this workflow is not appropriate to describe the precise activity of the wavefronts but only a surrogate of such activities. Although such CC are rigorous quantitative measurements of reconstruction accuracy; coarser information about singularity point locations is usually sufficient in clinical practice to guide the ablation strategy.

Results are better in the left atrium compared to the right atrium. Septal and posterior portions of the right atrium were especially error-prone in our simulations. This is due to a poor reconstruction of the epicardial signals at these locations. Reasons for this poor reconstruction may be due to the septum being deeply inserted in-between the two chambers, and the lateral right atrium being covered by the right pulmonary veins. These findings are consistent with previous clinical work findings are consistent with previous clinical work [2].

The main limitation of our study was the use of a homogeneous model for both the forward propagation and the inverse problem. However, a different formulation of the forward model was used for the simulation of body surface potentials (BEM) and the inverse problem (MFS), in addition to noise being added to the data.

\section{Acknowledgements}

This study was funded by the French Agence Nationale de la Recherche through the "Investissements d'Avenir” program (ANR-10-IAHU04-LIRYC).

\section{References}

[1] P. S. Cuculich, Y. Wang, et al., "Noninvasive Characterization of Epicardial Activation in Humans With Diverse Atrial Fibrillation Patterns / Clinical Perspective," Circulation, vol. 122, pp. 1364-1372, 2010.

[2] M. Haissaguerre, M. Hocini, et al., "Driver Domains in Persistent Atrial Fibrillation," Circulation, vol. 130, pp. 530-538, 2014.

[3] A. J. Shah, H. S. Lim, et al., "Noninvasive mapping of ventricular arrhythmias," Cardiac electrophysiology clinics, vol. 7, pp. 99-107, 2015.

[4] P. Kuklik, S. Zeemering, et al., "Identification of Rotors during Human Atrial Fibrillation using Contact Mapping and Phase Singularity Detection: Technical Considerations," IEEE Trans Biomed Eng, Apr 152016.

[5] J. Pedron-Torrecilla, M. Rodrigo, et al., "Noninvasive Estimation of Epicardial Dominant High-Frequency Regions During Atrial Fibrillation," J Cardiovasc Electrophysiol, vol. 27, pp. 435-42, Apr 2016.

[6] M. Rodrigo, M. S. Guillem, et al., "Body surface localization of left and right atrial high-frequency rotors in atrial fibrillation patients: A clinical-computational study," Heart Rhythm, vol. 11, pp. 1584-1591, 2014.

[7] Y. Wang and Y. Rudy, "Application of the method of fundamental solutions to potential-based inverse electrocardiography," Annals of Biomedical Engineering, vol. 34, pp. 1272-1288, 2006.

[8] P. Colli-Franzone, L. Guerri, et al., "A mathematical procedure for solving the inverse potential problem of electrocardiography. analysis of the time-space accuracy from in vitro experimental data," Mathematical Biosciences, vol. 77, pp. 353 - 396, 1985.

[9] J. Jalife, "Rotors and Spiral Waves in Atrial Fibrillation," Journal of Cardiovascular Electrophysiology, vol. 14, pp. 776-780, 2003.

[10] J. Lachaux, E. Rodriguez, et al., "Measuring Phase Synchrony in Brain Signals," Human Brain Mapping, vol. 8, 1999.

Address for correspondence.

Rémi Dubois

IHU LIRYC,

CHU Xavier Arnozan,

Ave du Haut Lévêque, 33600 Pessac, France

remi.dubois@espci.fr 